\title{
Thermodynamic and Conformational Properties of Styrene- Methyl Methacrylate Block Copolymers in Dilute Solution. VI. Chain Conformations Disclosed by Thin-Layer Chromatography
}

\author{
Fumio Kamiyama, ${ }^{*}$ Hiroshi InAgaki, ${ }^{* *}$ and Tadao Kotaka \\ Institute for Chemical Research, Kyoto University, Uji, Kyoto 611, Japan.
}

(Received September 16, 1971)

\begin{abstract}
The development characteristics in thin-layer chromatography was studied for block copolymers of BAB-type with equimolar composition but different molecular weights, where A and B are polystyrene (PS) and poly(methyl methacrylate) (PMMA), respectively. The values of $R_{\mathrm{f}}$ (rate of flow) obtained with a developer composed of nitroethane and acetone decreased with increasing molecular weight, whereas those with other developers, which behave as good solvents for both PS and PMMA, were little dependent on molecular weight. In view of our previous finding that the molecular weight dependence of $R_{\mathrm{f}}$ appears only when phase separation of sample polymer takes place during the development, the solution state of block copolymers in the solvent mixture of nitroethane and acetone was investigated by means of phase equilibrium and ultracentrifugation. No phase separation was observed. Thus a pertinent elucidation for the molecular weight dependence of $R_{\mathrm{f}}$ was given by taking into consideration that the random flight conformation of block copolymers collapses due to intramolecular phase separation between the block portions of PS and PMMA, which will be brought about because both solvents are nonsolvents for PS but good solvents for PMMA.

KEY WORDS Thin-Layer Chromatography / Phase Equilibrium / Velocity Ultracentrifugation / Styrene-Methacrylate Block Copolymers / Conformational Anomaly / Intramolecular Phase Separation /
\end{abstract}

Recently we have reported that block copolymers of a BAB-type, where A and B are polystyrene and poly(methyl methacrylate), respectively, exhibited an anomalous behavior in its hydrodynamic properties. $^{1}$ Intrinsic viscosities obtained in $p$-xylene for the block copolymers with high molecular weights were significantly lower than those for their precursor polystyrene samples in the same solvent. This result was argued to be indicative of collapse of the random flight chain conformation, ${ }^{* * *}$ particularly of two B-side blocks, due to the different solubility of the A- and B-block in p-xylene.

On the other hand, we have been engaged in

* Permanent address: Research Laboratory, Sekisui Chemical Co., Ltd., Mishima-gun, Osaka, Japan.

** To whom correspondence should be addressed.

***The meaning of this term used throughout this paper should be extended to pseudo-Gaussian conformations that chain polymers would assume due to the excluded volume effect between segments. searching for new applications of thin-layer chromatography (TLC) to polymer chemistry for nearly half a decade. This series of studies has yielded a variety of possibilities for determining compositional heterogeneity and monomer arrangement of copolymers, ${ }^{2,3}$ and for fractionating homopolymers into component species by the difference in steric isomerism ${ }^{4}$ and molecular weight, ${ }^{5}$ as well. Successful applications of TLC similar to ours were also reported by other authors. ${ }^{6}$

Some of these findings were interpreted in terms of a separation mechanism that involves an adsorption-desorption process between macromolecule and adsorbent in the presence of solvent molecule. ${ }^{7,8}$ For the state of the adsorption it has been speculated that polymeradsorbent interactions acting in this process should be concerned not with the chemical and/ or steric constitution averaged over a whole 
polymer chain but those which are assigned to some localized parts of the chain. ${ }^{9}$ Speculation allowed us to expect that the conformational change of block copolymers revealed by viscometry $^{1}$ may be further investigated by applying TLC, since some changes in the local constitution of polymer chain will be accompanied by collapse of the random flight conformation. Thus the present paper presents an additional evidence endorsing the comformational change in block copolymers by utilizing TLC techniques.

\section{EXPERIMENTAL}

\section{Polymer Samples}

Styrene (ST)-methyl methacrylate (MMA) block copolymers of BAB- and AB-type with nearly equimolar composition were used. The block copolymers of the former type were prepared by an anionic polymerization technique in tetrahydrofran (THF) at $-78^{\circ} \mathrm{C}$ with sodium biphenyl-THF solution as initiator. The details of preparation and characterization have been reported elsewhere. ${ }^{10}$ The block copolymer of AB-type was obtined also by an anionic polymerization technique with $n$-butyllithium- $n$ hexane solution as initiator. ${ }^{11}$ Besides these, ST-MMA statistical copolymers and a methyl methacrylate homopolymer (PMMA), which had been prepared for our previous studies, ${ }^{12}$ were used as reference samples. Table I summarizes the results of characterization of the polymer samples employed for the present experiment.

Table I. Characterization of sample polymers

\begin{tabular}{|c|c|c|c|}
\hline $\begin{array}{l}\text { Sample } \\
\text { code }\end{array}$ & $\begin{array}{l}\text { Monomer } \\
\text { arrangement }\end{array}$ & ST-mole $\%$ & $M_{w} \times 10^{-4}$ \\
\hline $25 \mathrm{~B}^{\mathrm{a}}$ & BAB & 51.9 & 26.0 \\
\hline $28 \mathrm{~B}^{\mathrm{a}}$ & BAB & 53.7 & 9.19 \\
\hline $43 B^{b}$ & $\mathrm{AB}$ & 50.0 & $6.80^{\mathrm{c}}$ \\
\hline $29 \mathrm{~B}^{\mathrm{a}}$ & BAB & 48.0 & 3.39 \\
\hline SM-1 & \multirow{4}{*}{ statistical } & 9.1 & - \\
\hline$S M-3^{c}$ & & 30.2 & 27.6 \\
\hline $\mathrm{SA}-3^{\mathrm{c}}$ & & 33.2 & $33.4^{\mathrm{d}}$ \\
\hline SM-5 & & 55.4 & 22.6 \\
\hline \multicolumn{4}{|c|}{$\begin{array}{l}\text { a ref } 10 . \quad \text { b ref } 11 . \quad \text { c ref } 12 . \\
\text { d Number-average value. } \\
\text { e The value of } M_{w} \text { was estimated from light scat- } \\
\text { tering data obtained in 2-butanone and toluene. } \\
\text { For the details of the practice, see ref } 1 .\end{array}$} \\
\hline
\end{tabular}

\section{TLC Technique}

The procedure of TLC has been described elsewhere in detail. ${ }^{2-5}$ A silica gel, Kilselgel G (Merck AG., Darmstadt, Germany), was used as adsorbent. The thickness of thin-layer was adjusted to $0.3 \mathrm{~mm}$. Polymer samples were dissolved in trichloroethylene at $c a .0 .5 \mathrm{~g} / \mathrm{d} l$ for stock solution. Usual ascending and concentration gradient development techniques were employed alternatively corresponding to each experimental purpose. When the latter technique was applied, an additional solvent was admixed to an initial solvent in such a way the rate of addition is kept rapid at the very beginning but decreased gradually with elevation of the solvent front. Chromatograms were stained with 1-\% methanol solution of iodine.

\section{Ultracentrifugation}

To examine if the block copolymer samples exhibit phase separation in some solvent mixtures used as developer, velocity ultracentrifugation experiments were made on a Hitachi analytical ultracentrifuge UCA-1A furnished with a schlieren optics.

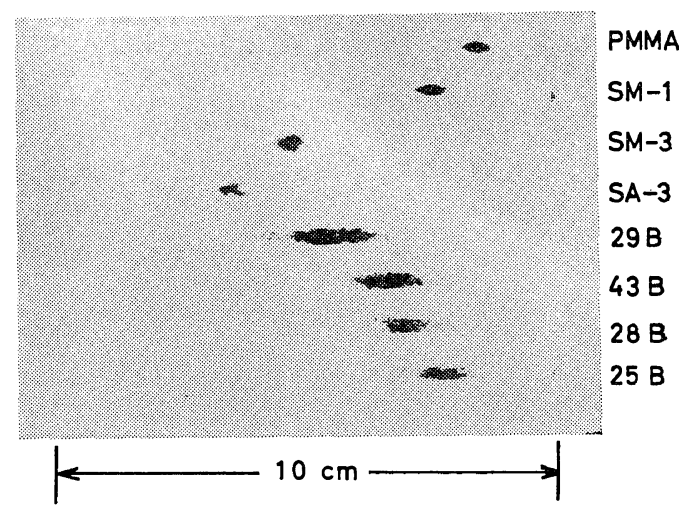

Figure 1. TLC chromatogram obtained for STMMA block and statistical copolymers with a solvent system nitroethane and acetone as developer.

\section{RESULTS}

Figure 1 shows a chromatogram obtained for the block copolymers with nearly equimolar composition but different molecular weights ranging from 34000 to 260000 . Samples of ST-MMA statistical copolymers with different 
compositions, and PMMA were developed simultaneously on the same chromatoplate. A concentration gradient technique with a developer system nitroethane +acetone, which behaves as poor solvent toward the block copolymers, was applied. The development was started with 40 $\mathrm{m} l$ of nitroethane as the initial solvent and completed by adding $15 \mathrm{~m} l$ of acetone. Inspection of the chromatogram reveals that the block copolymers migrate more slowly with increasing MMA-content. Further inquiries were made into whether or not the developer system allows migration of statistical copolymers with the same composition at the same rate independent of molecular weight. The result is shown in Table II, which indicates that the values of rate of flow $\left(\boldsymbol{R}_{\mathrm{f}}\right)$ depend little on molecular weight, and this is in agreement with those obtained previously in so far as the development proceeds according to adsorption-desorption process (adsorption chromatography). ${ }^{2-4,7,8}$

Table II. Values of $R^{\mathrm{f}}$ obtained for ST-MMA statistical copolymers with solvent system nitroethane and acetone as developer

\begin{tabular}{cccc}
\hline $\begin{array}{c}\text { Somple } \\
\text { code }^{\mathrm{a}}\end{array}$ & ST-mole $\%$ & $M_{w} \times 10^{-4}$ & $R_{\mathrm{f}}$ \\
\hline SM-3-1 & 29.9 & 105.8 & 0.33 \\
SM-3-5 & 28.6 & 77.4 & 0.34 \\
SM-3-9 & 30.2 & 27.6 & 0.35
\end{tabular}

${ }^{a}$ For the preparation and characterization, see ref 12.

On the other hand, it has been well recognized that the molecular weight dependence of $\boldsymbol{R}_{\mathrm{f}}$ appears only when the development is dominantly dictated by a process of phase separation of sample polymer on chromatoplate. ${ }^{5-7}$ In this connection, a separate experiment on phase equilibrium was made. Sample 25B with the highest molecular weight of those used was dissolved at $0.3 \mathrm{~g} / \mathrm{d} l$ in nitroethane or a mixture nitroethone +acetone with a composition of $8: 3$ by volume, which corresponded to that finally attained in the TLC experiment. No trend of phase separation was observed at room temperature though the solutions gave rise to visibly turbid solutions.

To further investigate the dispersed state of

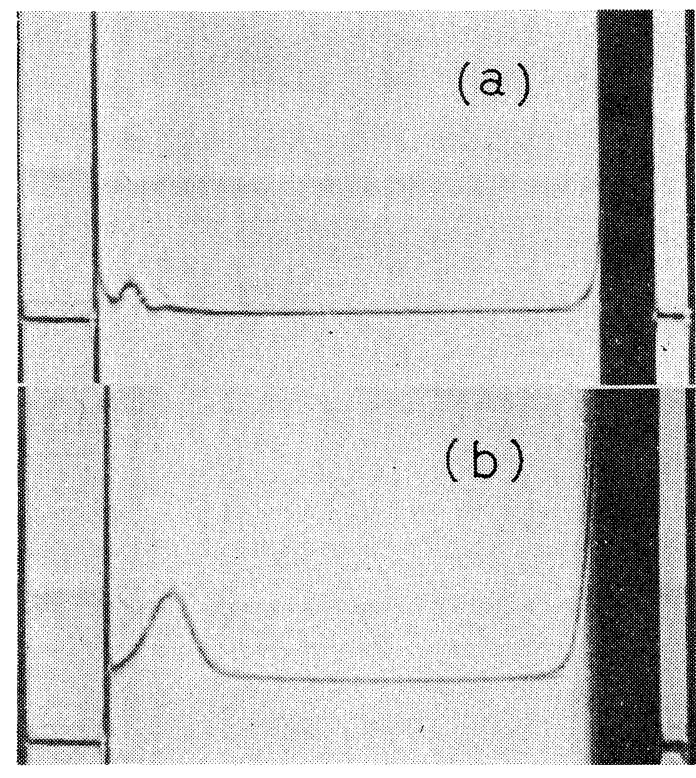

Figure 2. Sedimentation patterns (a) and (b) for block copolymer 25B dissolved in a solvent system nitroethane and acetone ( $8: 3$ by volume) taken at $8950 \mathrm{rpm}$ and after $43700 \mathrm{rpm}$ was attained, respectively.

25B in the solvent mixture, the solutions of different concentrations, i.e., 0.40 and $0.80 \mathrm{~g} / \mathrm{d} l$, were subjected to velocity ultracentrifugation. By way of example, the sedimentation pattern first taken at a rotor speed of $8950 \mathrm{rpm}$ and that taken after $43700 \mathrm{rpm}$ was attained are shown in Figures 2 (a) and (b), respectively. The weight fraction of the fast sedimenting component estimated from the patterns was $c a$. 0.13. This indicates that gel-like aggregates were involved only in a quite small portion, and no phase separation took place. Thus we may conclude, at least, that the block copolymers were not developed according to the separation mechanism in which phase separation process is operative..$^{5,7}$

In relation with the above conclusion we made other TLC experiments on the block and statistical copolymers by using different developers, namely solvent systems benzene +2 -butanone, and carbon tetrachloride + methyl acetate. The former developer behaves as a good solvent for both polystyrene (PS) and PMMA. For the latter, carbon tetrachloride is a good solvent for PS but a poor solvent for PMMA; while methyl 
acetate is just opposite. Consequently a mixture of these solvents will be moderately good for the block copolymer, and better than the system nitroethane+acetone but poorer than the former. Figure 3 shows the chromatogram obtained by applying a concentration gradient technique with $30 \mathrm{~m} l$ of benzene and $25 \mathrm{ml}$ of 2-butanone as the initial and additional solvent, respectively. An analogous result to Figure 3 was observed with the latter system and is given in Table III together with those obtained by other developers. These results indicate that the molecular weight dependence of $R_{\mathrm{f}}$ almost disappears when the developer is a good or moderately good solvent for polymer.



PMMA

$S M-1$

$S M-3$

$S M-5$

$29 B$

$43 \mathrm{~B}$

$28 \mathrm{~B}$

$25 B$

Figure 3. TLC chromatogram obtained for block and statistical copolymers with a solvent system benzene and 2-butanone as developer.

\section{DISCUSSION}

At the beginning of this section, we present some rules of the development characteristics of copolymers which have been deduced from our previous studies. ${ }^{2-5,7-9}$

Rule [1]: $R_{\mathrm{f}}$-values of copolymers composed of a given comonomer pair decrease with an increase in the content of more polar unit of the comonomer pair but are little dependent on the molecular weight. ${ }^{2}$ Rule [2]: $R_{\mathrm{f}}$-values of copolymers composed of a given comonomer pair with the same composition can be different if the monomer arrangement along the chain differs from one another, but are little dependent on the molecular weight; ${ }^{3,9}$ this holds for homopolymers with difierent steric isomerism. ${ }^{4}$ Rule [3]: Distinct molecular weight dependences of $R_{\mathrm{f}}$ in a range of molecular weights higher than ca. $5 \times 10^{4}$ appear only when phase separation of sample polymer takes place during the development but does not in adsorption chromatography; ${ }^{5-7}$ in other words, the origin of the molecular weight dependence of $\boldsymbol{R}_{\mathrm{f}}$ is related closely to the solubility of sample polymers in solvents used as the developer.

First we should point out that rule [2] could be explained by presuming that interactions between polymer and adsorbent at solid-liquid interfaces are concerned with the chemical and/ or steric constitution of some localized parts of the polymer chain, e.g., dyads, triads, and so on. ${ }^{3,4,9}$ In fact, the observation that the $R_{\mathrm{f}}$ value found for an equimolar ST-MMA (or -MA) statistical copolymer was significantly higher than that for an alternating copolymer of the same comonomer has been interpreted in terms of a dyad model., ${ }^{3,9}$ By this model it was meant that the adsorptive force of a ST-MMA copolymer chain onto adsorbent, $F_{\mathrm{c}}$, could be approximated by

$$
F_{\mathrm{c}}=P_{\mathrm{ss}} F_{\mathrm{ss}}+P_{\mathrm{sm}} F_{\mathrm{sm}}+P_{\mathrm{mm}} F_{\mathrm{mm}},
$$

where $F$ 's are the adsorptive force assigned to each dyad, i.e., ST-ST, ST-MMA, and MMA -MMA; $P$ 's are the probability that a given dyad is found in the copolymer chain; hence, this is related to the monomer arrangement. However, the result that this model was valid implicates that each dyad along the polymer chain should, in equal probability, be able to make contact with adsorbent. Therefore the condition required for the validity of the model will not be fulfilled if the random flight conformation is collapsed for some reason: in such a case, the development characteristics of copolymers will be no longer effected by the difference in the monomer arrangement but by the density of each dyad or monomer unit on the surface of spatial domain occupied by the collapsed polymer chain, or simply speaking, by the shape of collapsed polymer chain.

From the above argument it may be expected that a pertinent elucidation for the molecular weight dependence of $R_{\mathrm{f}}$ is given by considering the conformation which the block copolymer 
chain would assume in the mixture nitroethane +acetone. Since both solvents are nonsolvents for PS but good solvents for PMMA, the conformation of block copolymer will be more and more collapsed with increasing molecular weight so that intramolecular phase separation takes place. Such a deformed conformation may be described by a model in which the block portion of PS is tightly coiled up and wrapped by those of PMMA - the segregated chain model. This model allows us to predict that with respect to the density of MMA-units on the surface of spacial domain occupied by a polymer chain, the block copolymer with a high enough molecular weight becomes indistinguishable from PMMA. This means, in turn, that at the limit of infinite molecular weight, values of $R_{\mathrm{f}}$ expected for the block copolymers with the developer system nitroethane+acetone should approach a value of $R_{\mathrm{f}}$ obtained for PMMA under the same development condition.

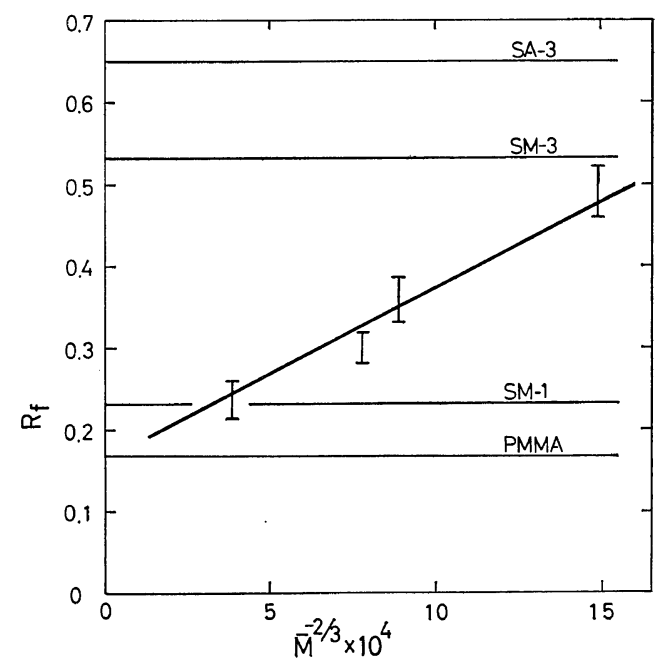

Figure 4. Plot of $R_{\mathrm{f}}$-values evaluated from Figure 1 against $\bar{M}^{-2 / 3}, \bar{M}$ being the geometric mean of molecular weights of each block portion. For the details, see text.

To examine whether or not the above prediction can be justified, the $R_{\mathrm{f}}$-values determined from Figure 1 are plotted against $\bar{M}^{-2 / 3}$ in Figure 4, where $\bar{M}$ is the geometric mean of molecular weights of each block portion. This plot was constructed, however, only by an intuitive rea- son that the intramolecular phase separation might be related to the phase equilibrium in a ternary system, two polymers-one solvent. ${ }^{1,13,14}$ Lines drawn parallel to the abscissa in the Figure indicate levels of migration to which the statistical copolymers and PMMA were developed in the simultaneous experiment with the block copolymers. The plot may be approximated by a straight line, and extrapolation of the line to infinite molecular weight reaches a point, which may be identified with the $R_{\mathrm{f}}$-value for PMMA. Thus our prediction made according to the segregated chain model was justified.

Before discussing the increase of $R_{\mathrm{f}}$ with decreasing molecular weight shown in Figure 1, let us recall rule [1], which means, for the case of ST-MMA statistical copolymers, that the $R_{\mathrm{f}}$-value increases with increasing ST-content. Taking this rule into account, we may expect that the origin of the increase of $R_{\mathrm{f}}$ with decreasing molecular weight of the block copolymer is attributable to the increase in the density of ST-units on the surface of polymer domain. This must be quite conceivable because the segregated chain conformation will more and more revert to that of the random flight as the molecular weight decreases. This is expected also from the experimental fact that two polymers with different chemical constitution become more and more compatible as their molecular

Table III. Summary of TLC results obtained with different developer systems ${ }^{a}$

\begin{tabular}{cccc}
\hline \multirow{2}{*}{ Sample code } & \multicolumn{3}{c}{$R_{\mathrm{f} \text {-values }}$} \\
\cline { 2 - 4 } & $(\mathrm{i})$ & $(\mathrm{ii})$ & $(\mathrm{iii})$ \\
\hline 29B & 0.49 & 0.60 & 0.54 \\
43B & 0.36 & 0.54 & 0.53 \\
28B & 0.30 & 0.54 & 0.53 \\
25B & 0.24 & 0.53 & 0.49 \\
SM-5 & - & - & 0.74 \\
SA-3 & 0.65 & 0.9 & - \\
SM-3-9 & 0.53 & 0.83 & 0.67 \\
SM-1 & 0.23 & 0.42 & 0.31 \\
PMMA & 0.17 & 0.26 & 0 \\
\hline Resolving power & 0.06 & 0.16 & 0.31 \\
of developer & & & \\
\hline
\end{tabular}

a (i), nitroethane+acetone; (ii), carbon tetrachloride + methyl acetate; (iii), benzene + butanone.

b For the definition, see text. 
weights decrease. ${ }^{13}$ Moreover the molecular weight dependence of $R_{\mathrm{f}}$, in fact, diminished in the experiments made with the developer systems which behave as good or moderately good solvents for the block copolymers, as Figure 3 and Table III indicate.

Last to be mentioned concerns the resolving power of developer, which indicates how far apart two given different samples can be separated on chromatogram. Needless to say, but this is very important for a comparative discussion of the molecular weight dependences of $\boldsymbol{R}_{\mathrm{f}}$ found for the block copolymers with different developer systems, because the $R_{\mathrm{f}}$-value is different for different developer. For our purpose we shall approximate the resolving power of each developer by the difference between $R_{\mathrm{f}^{-}}$ values obtained for samples PMMA and SM-1. The values are listed on the last row of Table III, and this result proves that despite its low resolving power, the molecular weight dependence of $R_{\mathrm{f}}$ found with the mixture nitroethane +acetone is apparently strong in comparison with those obtained with the other developer systems.

On the basis of the above investigation it is concluded that in good solvents of mixtures for both parent homopolymers, the block copolymers assume the random flight chain conformation, whereas in selective solvents or mixtures, they assume a deformed conformation as pictured by the segregated chain model. This conclusion is, of course, in agreement with that drawn from our previous works on the hydrodynamic and other behaviors. ${ }^{1,14,15}$ From the standpoint of TLC applied to polymer chemistry it is pointed out that a new type of the development characteristics in adsorption chromatography was found which is specified by the distinct molecular weight dependence of $R_{\mathrm{f}}$ in the range of molecular weights higher than $c a .5 \times 10^{4}$. This finding might suggest, in turn, a possibility that conformational changes of macromolecules are detected by applying TLC techniques.
Acknowledgment. One of the present authors (F. K.) thanks Dr. N. Sagane, Director of Research Laboratory, Sekisui Chemical Co., Ltd., for kindly delegating him to this Institute.

\section{REFERENCES}

1. H. Ohnuma, T. Kotaka, and H. Inagaki, Polymer J., 1, 716 (1970).

2. H. Inagaki, H. Matsuda, and F. Kamiyama, Macromolecules, 1, 520 (1968).

3. F. Kamiyama, H. Matsuda, and H. Inagaki, Makromol. Chem., 125, 286 (1969).

4. H. Inagaki, T. Miyamoto, and F. Kamiyama, J. Polym. Sci., Part B, 7, 329 (1969); T. Miyamoto, and $\mathrm{H}$. Inagaki, Macromolecules, 2, 544 (1969).

5. F. Kamiyama, H. Matsuda, and H. Inagaki, Polymer J., 1, 518 (1970).

6. V. G. Belenkii and E. S. Gankia, Dokl. Akad. Nauk SSSR, 186, 857 (1969); V. G. Belenkii and E. S. Gankina, J. Chromatogr., 53, 3 (1970); E. P. Otocka, and M. Y. Hellman, Macromolecules, 3, 362 (1970); E. P. Otocka, ibid., 3, 691 (1970).

7. H. Inagaki, F. Kamiyama, and T. Yagi, ibid., 4, 133 (1971).

8. F. Kamiyama, and H. Inagaki, Bull. Inst. Chem. Res., Kyoto Univ., 49, 53 (1971).

9. H. Inagaki, ibid., 47, 196 (1969).

10. H. Ohnuma, T. Kotaka, and H. Inagaki, Polymer, 10, 501 (1969).

11. T. Kotaka, T. Tanaka, and H. Inagaki, Polymer J., 3, 327 (1972).

12. T. Kotaka, Y. Murakami, and H. Inagaki, $J$. Phys. Chem., 72, 829 (1968).

13. D. Berek, B. Böhmer, and D. Lath, Plaste. Kautschuk, 14, 556 (1967).

14. T. Kotaka, H. Ohnuma, and H. Inagaki, Polymer, 10, 517 (1969); T. Kotaka, H. Ohnuma, and H. Inagaki, "Dilute Solution Behavior of Block Copolymers" in "Colloidal and Morphological Behavior of Block and Graft Gopolymers" G. E. Molau, Ed., Plenum Press, New York-London, 1971, pp 259-277.

15. T. Tanaka, T. Kotaka, and H. Inagaki, Polymer J., 3, 338 (1972). 